\title{
Amyloid $\beta$ oligomers elicit mitochondrial transport defects and fragmentation in a time-dependent and pathway-specific manner
}

\author{
Yanfang Rui ${ }^{1,3}$ and James Q. Zheng ${ }^{1,2,3^{*}}$
}

\begin{abstract}
Small oligomeric forms of amyloid- $\beta$ (A $\beta$ ) are believed to be the culprit for declined brain functions in AD in part through their impairment of neuronal trafficking and synaptic functions. However, the precise cellular actions of $A \beta$ oligomers and underlying mechanisms in neurons remain to be fully defined. Previous studies have identified mitochondria as a major target of $A \beta$ toxicity contributing to early cognitive decline and memory loss in neurodegenerative diseases including Alzheimer's disease (AD). In this study, we report that A oligomers acutely elicit distinct effects on the transport and integrity of mitochondria. We found that acute exposure of hippocampal neurons to $A \beta$ oligomers from either synthetic peptides or AD brain homogenates selectively impaired fast transport of mitochondria without affecting the movement of late endosomes and lysosomes. Extended exposure of hipoocampal neurons to $A \beta$ oligomers was found to result in mitochondrial fragmentation. While both mitochondrial effects induced by A $A$ oligomers can be abolished by the inhibition of GSK3 $\beta$, they appear to be independent from each other. A $\beta$ oligomers impaired mitochondrial transport through HDAC6 activation whereas the fragmentation involved the GTPase Drp-1. These results show that A $\beta$ oligomers can acutely disrupt mitochondrial transport and integrity in a time-dependent and pathway-specific manner. These findings thus provide new insights into Aß-induced mitochondrial defects that may contribute to neuronal dysfunction and AD pathogenesis.
\end{abstract}

Keywords: Alzheimer's disease, Transport, Fragmentation, Hippocampus, HDAC6, Drp-1, GSK3 $\beta$

Abbreviations: $A \beta$, Amyloid- $\beta$; $A \beta-O, A \beta$ oligomers; $C C D$, Charge coupled device; GSK3 $\beta$, Glycogen synthase kinase $3 \beta$; HDAC, Histone deacetylase; IP, Immunoprecipitation; NA, Numeric aperture; TSA, Trichostatin A

\section{Introduction}

Alzheimer's disease (AD) is a progressive neurodegenerative brain disorder that is characterized by two hallmarks: intracellular neurofibrillary tangles and extracellular amyloid- $\beta(A \beta)$ plaques in the regions of the brain that are responsible for learning and memory. $A \beta$, peptides of 39-43 amino acids produced by the sequential cleavage of $\beta$ - and $r$-secretase at the $C$ terminal of amyloid precursor protein, are believed to play a central role in the

\footnotetext{
* Correspondence: james.zheng@emory.edu

'Department of Cell Biology, Emory University School of Medicine, 615 Michael Street, Atlanta, GA 30322, USA

2Department of Neurology, Emory University School of Medicine, Atlanta, GA 30322, USA

Full list of author information is available at the end of the article
}

neurodegeneration and subsequent cognitive abnormalities of AD brains [1]. Recent evidence indicates that small oligomeric species of $A \beta$ are responsible for impaired brain functions in the early stages of $A D$, in part through their detrimental actions on neuronal trafficking and synaptic functions [2-5]. Increasing evidence suggests that mitochondrial abnormalities play a critical role in $\mathrm{AD}$ pathogenesis. However, our understanding of various $A \beta$ effects on mitochondria and their contribution to AD brain's dysfunction and disease progression remains incomplete.

Mitochondria are a cell's vital organelle of energy production and they participate in diverse cellular functions ranging from $\mathrm{Ca}^{2+}$ homeostasis to cell apoptosis. Mitochondria are mainly produced in the soma and transported 
to the specific subcellular regions via microtubule (MT)-based fast transport [6, 7]. In neurons, mitochondria are seen accumulated at the pre- and post-synaptic sites where they function in synaptic transmission and plasticity [8-12]. Such subcellular localization of mitochondria involves spatiotemporal regulation of MT-based motors as well as interactions with other cytoskeletal structures such as the actin cytoskeleton and intermediate filaments [6]. The half-life of mitochondria is about 1 month and mitochondrial fusion and fission provide a mechanism for mitochondrial renewal and degradation $[10,13,14]$. Therefore, timely and proper delivery of new mitochondria from the cell body is important to support the health and function of mitochondria in neurons.

Disruption of mitochondrial transport, structure, and function is believed to be a major contributor to many neurodegenerative diseases $[10,15]$. Interestingly, disrupted axonal transport has been observed prior to the major pathological hallmarks such as senile plaques in AD $[16,17]$. In many of these cases, axons exhibit swellings and varicosities with accumulated mitochondria and other organelles. On the other hand, damaged mitochondria dynamics in dendrite has been associated with the synaptic deficit in $\mathrm{AD}[18,19]$. Mitochondria that are stuck in the swellings/varicosities often look unhealthy and appear to undergo degradation and fragmentation. The cellular mechanisms that lead to defected mitochondrial transport and structure remain to be fully defined. In this study, we utilized cultured hippocampal neurons to investigate the acute effects of small $A \beta$ oligomers on mitochondria. In particular, we examined the effects of $\mathrm{A} \beta$ oligomers prepared from synthetic peptides and the human AD brain homogenates on mitochondrial transport and morphology. Our data show that $A \beta$ oligomers generated two distinct effects on mitochondria that are temporally segregated: transport impairment preceded the fragmentation. We further investigated the underlying mechanisms that mediate the specific effects of small $A \beta$ oligomers on mitochondria.

\section{Methods}

\section{Hippocampal culture, transfection and live imaging}

All the experiments involving the use of vertebrate animals were carried out in accordance to the NIH guideline for animal use and have been approved by the Institutional Animal Care and Use Committee of Emory University. Hippocampal neurons from embryonic day 18 rats were obtained as described previously [20]. About $\sim 200,000$ cells were plated in a $35 \mathrm{~mm}$ glass bottom culture dish (Warner Instruments, Hamden, CT) that were coated with $100 \mu \mathrm{g} / \mathrm{ml}$ poly-D-lysine (Sigma, St. Louis, MO). Hippocampal neurons were cultured in the Neurobasal medium containing B27 and Glutamax (Invitrogen) and typically imaged after 3-7 days in culture. Transfection of hippocampal neurons was performed using the CalPhos Mammalian Transfection Kit (Clontech, Mountain View, CA), which was typically done one day before the imaging [19]. The DNA constructs for transfection were prepared by plasmid maxi kit (Qiagen, Valencia, CA). Mito-DsRed and Mito-GFP were provided by Dr. Zheng $\mathrm{Li}$ at NIH/ NIMH. All the labeling and imaging were carried out in Krebs'-Ringer's buffer (KRB, in mM: $150 \mathrm{NaCl}, 5 \mathrm{KCl}, 2$ $\mathrm{CaCl}_{2}, 1 \mathrm{MgCl}_{2}, 10$ glucose, and 10 HEPES, pH 7.4) $[19,21,22]$. For simultaneous imaging of mitochondria and lysosome trafficking, neurons were sequentially labeled by MitoTracker Green (20 nM for $20 \mathrm{~min}$; Invitrogen, Eugene, OR) and LysoTracker Red (100 nM for $30 \mathrm{~min}$; Invitrogen), followed by three rinses and additional 10 min incubation at $37^{\circ} \mathrm{C}$ before imaging.

Dual-channel fluorescent time-lapse recordings were performed on an inverted microscope (TE2000, Nikon) equipped with a multispectral imager (Dual-View; Optical Insights) using either a $40 \times$ with 1.3 numeric aperture (NA) S Fluor oil immersion objective or a $60 \times 1.4 \mathrm{NA}$ Plan Apo oil immersion objective with identical settings between the control and experimental groups. Time-lapse images were captured with a charge-coupled device (CCD) camera (SensiCam QE, Cooke Scientific) using the IPLab imaging software (BD Biosciences). We typically recorded neurons at a sampling rate of one frame every $5 \mathrm{~s}$ for $5 \mathrm{~min}$, with the CCD exposure at $50 \mathrm{~ms}$ and $2 \times 2$ binning. For each experiment, a population of neurons was imaged for a $5 \mathrm{~min}$ control period before the application of $\mathrm{A} \beta$ molecules, followed by another 5 min time-lapse recording after $A \beta$ exposure for $30 \mathrm{~min}$ or $2 \mathrm{~h}$. All of the experiments were performed on the microscope stage with the $35 \mathrm{~mm}$ dish housed in a temperature controlled chamber (Warner Instruments, New Haven, CT) with the temperature set at $\sim 35{ }^{\circ} \mathrm{C}$. For different inhibitors, we applied the inhibitor $20 \mathrm{~min}$ before the onset of $A \beta$ exposure. Tubacin was provided by Dr. Stuart Schreiber at Broad Institute of Harvard and MIT through the support by the Initiative for Chemical Genetics, National Cancer Institute. SB415286 and SB216763 were purchased from Tocris Bioscience (Ellisville, MO), Trichostatin A (TSA) was from Sigma (St. Louis, MO), and MS-275 was from Selleckchem (Houston, TX).

We quantified the $A \beta$ effects on organelle movement by determining the percentage of moving mitochondria before and after $A \beta$ exposure $[19,22]$. Here, quantification was done by repeatedly playing back the time-lapse sequences and counting the number of moving mitochondria or other organelles (moved $>3 \mu \mathrm{m}$ ) in each $5 \mathrm{~min}$ time-lapse sequence. We normalized the number of moving mitochondria or other organelles in the 5-min sequence against that before the $A \beta$ application. A value of $100 \%$ indicates that same numbers of moving 
mitochondria or vesicles were observed in both recording periods $[19,22]$. To generate the movement traces of mitochondria and other organelles, ImageJ (National Institutes of Health, Bethesda, MD) was used to first process the image sequence using the Zprojection function (maximum intensity), followed by division against the first frame to produce the final image of the moving traces [22]. The movement traces were also used to confirm the number of moving mitochondria over the 5 min time-lapse. Kymograph analysis was done using ImageJ with the multiple kymograph plugin written by J. Rietdorf (FMI Basel) and A. Seitz (EMBL Heidelberg). Here, Zprojection of the time-lapse sequence was used to identify the moving organelles and segmented line regions of interest (ROIs) were created from the movement traces. These segmented line ROIs were then applied to the time-lapse sequence to produce kymographs that allow the measurement of the average speed of each moving organelle.

\section{$A \beta$ oligomeric preparation and treatment}

$A \beta$ oligomers were prepared from either synthetic $A \beta_{1-42}$ (American Peptide Company Inc, Sunnydale,CA) or recombinant $A \beta_{1-42}$ (r-Peptide Company, Bogart, GA) according to the procedure described previously [23]. In brief, $\mathrm{A} \beta_{1-42}$ was dissolved in hexafluoro-2-propanol (HFIP) and aliquoted to microcentrifuge tubes. HFIP was subsequently removed by evaporation in a speed-vacuum and desiccated $\mathrm{A} \beta$ aliquots were stored at $-20^{\circ} \mathrm{C}$. To make $\mathrm{A} \beta$ oligomers, $\mathrm{A} \beta_{1-42}$ was dissolved in DMSO to make a $5 \mathrm{mM}$ stock solution. The stock solution was then diluted to $100 \mu \mathrm{M}$ with KRB and kept at $4{ }^{\circ} \mathrm{C}$ for $24 \mathrm{~h}$ before use. Bath application of $A \beta$ was achieved through a two-step dilution procedure. First, the $A \beta$ stock solution was diluted in KRB to twice the designated concentration $(2 \times$ working stock). The $2 \times$ working stock solution was warmed to $\sim 35{ }^{\circ} \mathrm{C}$ and then gently added to and mixed with the bath saline of the cells in an equal volume to reach the desired final concentration. In a typical experiment, $1 \mathrm{ml}$ of the $2 \times$ stock solution was added to $1 \mathrm{ml}$ of the bath solution in the culture/imaging dish on the microscope stage.

Postmortem human brain tissues were obtained from the tissue bank of the Emory Alzheimer's Disease Research Center and the Emory Center for Neurodegenerative Disease. Frozen tissues from the frontal cortex of age- and gender-matched control $(n=3)$ and $\mathrm{AD}(n=3)$ subjects were provided. Control subjects had no clinical history of neurological disease and were free of neurodegenerative disease pathology at autopsy. AD subjects met both CERAD [24] and NIA-Reagan [25] criteria for the neuropathologic diagnosis of $\mathrm{AD}$. The frozen tissues were weighted and homogenized in ice-cold phosphate-buffered saline at $20 \mathrm{~g} / 100 \mathrm{ml}$ using a Dounce homogenizer (A size pestle for 15 strokes, B size pestle for 15 strokes).
Homogenate was then sonicated 3 times for $5 \mathrm{~s}$ (power around 4), vortexed, and centrifuged for $5 \mathrm{~min}$ at $3000 \mathrm{~g}$. The supernatant was collected, aliquoted in eppendorf tubes, and stored at $-80{ }^{\circ} \mathrm{C}$. Before application to the cells, each aliquot was thawed and centrifuged at 10,000 rpm for $5 \mathrm{~min}$ to remove any aggregates and precipitates. Similar to the application of $A \beta$ oligomers from synthetic peptides, we applied the soluble human brain homogenate through a two-step dilution procedure: the $2 \times$ working stock and then 1:1 (volume:volume) to the cells.

Silver staining, western blotting and immunoprecipitation A $\beta$ samples (200 ng each) from American Peptide Company or $\mathrm{r}$-Peptide Company were loaded to sample buffer with $50 \mathrm{mM}$ DTT and heated at $85{ }^{\circ} \mathrm{C}$ for $5 \mathrm{~min}$. Samples were loaded and fractioned by SDS-PAGE on 10-20\% Tris-Tricine gel (Invitrogen) for $90 \mathrm{~min}$. After electrophoresis, the gel was briefly rinsed with ultrapure water and subjected to silver staining using SilverXpress ${ }^{\circ}$ Silver Staining Kit (Invitrogen). For western blotting, different $A \beta$ samples from synthetic peptides or human brain tissues were loaded in equal volume and fractioned by SDS-PAGE using 10$20 \%$ Tris-Tricine gel and subsequently transferred to nitrocellulose membrane. The membrane was boiled for $10 \mathrm{~min}$ in PBS and blocked with $5 \%$ non-fat dry milk in TBS with $0.05 \%$ Tween- 20 for $1 \mathrm{~h}$ at room temperature. The membrane was then incubated with a mixture of two anti-A $\beta$ antibodies: 6E10 (1:1000) and 4G8 (1:1000) antibodies (Signet, Dedham, MA) in blocking buffer overnight at $4{ }^{\circ} \mathrm{C}$. Bound antibodies were detected by HRP-conjugated secondary antibody, visualized by chemiluminescence using ECL (Thermo Scientific, Rockford, IL), and quantified using the gel analysis routine of ImageJ software (NIH).

To deplete $A \beta$ molecules from the soluble human brain homogenate, we performed immunoprecipitation (IP) using Protein A-agarose beads (Santa Cruz). Here, the homogenate samples were incubated with $10 \mu \mathrm{l}(2 \mu \mathrm{g})$ polyclonal anti-A $\beta$ antibody A8326 (Sigma) for $1 \mathrm{~h}$ at $4{ }^{\circ} \mathrm{C}$, followed by incubation with Protein A-agarose beads at $4{ }^{\circ} \mathrm{C}$ overnight on a rocker platform. The samples were subjected to centrifugation at $2500 \mathrm{rpm}$ for $5 \mathrm{~min}$ at $4{ }^{\circ} \mathrm{C}$ to allow the collection of the supernatant (referred to as Thru 1st) and pellet (referred to as Output 1st) respectively. Parts of the supernatant from the first round IP (Thru 1st) was used to test the effects on organelle trafficking. Another round of IP was done with the remaining supernatant to obtain the second round IP supernatant (Thru 2st) and pellet (Output 2st). All these samples after IP were examined by western blot as described above.

\section{Results}

$A \beta$ oligomers rapidly impair mitochondrial transport We double labeled mitochondria and late endosomes/ lysosomes of cultured hippocampal neurons with 
MitoTracker and LysoTracker, and examined the effects of $A \beta$ oligomers on the movement of these organelles $[19,22]$. We first tested $A \beta$ oligomers prepared from synthetic $A \beta_{1-42}$ and both western blotting and silver staining confirmed the presence of small $A \beta$ oligomers, including dimers, trimers, and tetramers, in this $A \beta$ oligomeric preparation from synthetic $A \beta_{1-42}$ (hereafter referred to as $A \beta-O$; Fig. 1a). A $\beta-O$ also contained a large portion of monomers that exert no effect on mitochondrial transport as we showed before [19]. We estimated from different batches of $\mathrm{A} \beta-\mathrm{O}$ that the small oligomers account for $\sim 10 \%$ of the total $A \beta$ concentration [19]. We found that exposure of cultured hippocampal neurons to $A \beta-O(1 \mu \mathrm{M}$ total $A \beta, \sim 100 \mathrm{nM}$ of oligomers) markedly inhibited the fast transport of mitochondria without affecting late endosomes/lysosomes (hereafter referred to as endo/lysosomes) of the same neurons, as evidenced by the movement tracings from the 5-min time-lapse sequences (Fig. 1b). To quantitatively determine the effects of $\mathrm{A} \beta-\mathrm{O}$ on the transport of

\section{a}
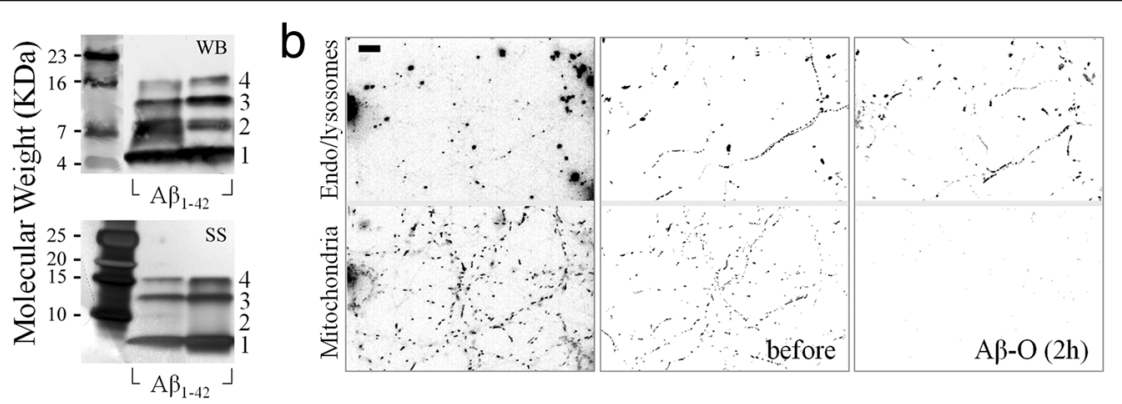

C
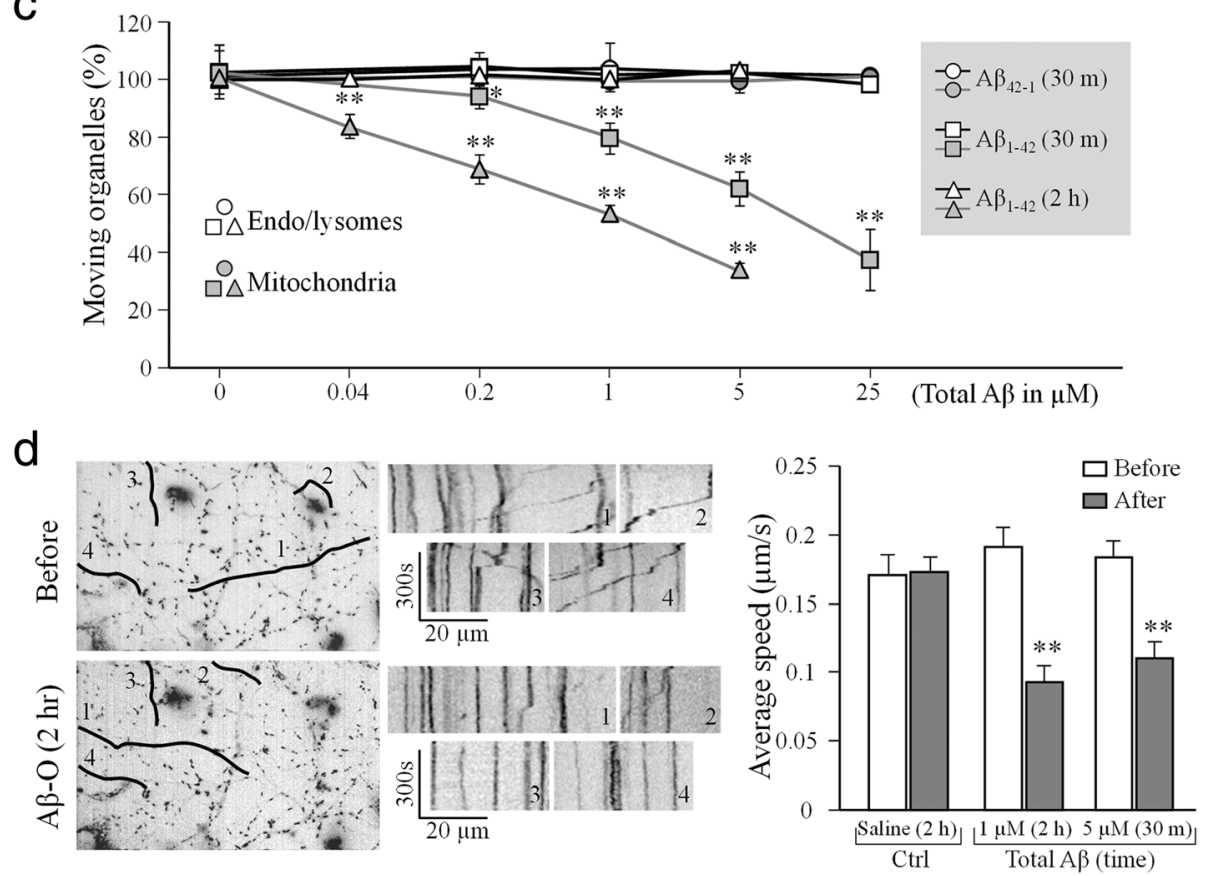

Fig. 1 Selective inhibition of fast transport of mitochondria by A oligomers. a Western blotting (WB, upper panel) and silver staining (SS, lower panel) showing the presence of $A \beta$ monomers (1) and small $A \beta$ oligomers, including dimmers (2), trimmers (3), and tetramers (4) in our $A \beta$ oligomeric preparation ( $A \beta-O)$ from synthetic $A \beta_{1-42}$. b Representative images showing hippocampal neurons with their late endosomes/lysosomes (endo/lysosomes; upper panels) and mitochondria (lower panels) labeled by LysoTracker and MitoTracker (left panels), as well as their movement tracings generated from 5 min time-lapse sequences before (middle panels) and after (right panels) $2 \mathrm{~h}$ exposure to $1 \mu \mathrm{M} \mathrm{A} \beta-\mathrm{O}$. Scale bar: $10 \mu \mathrm{m}$. c Quantitative analysis showing the dose- and exposure time-dependent $\mathrm{A} \beta-\mathrm{O}$ effects on the fast transport of mitochondria and endo/lysosomes. $A \beta_{42-1}$ was the negative control. All the data were normalized to the number of moving organelles in the 5-min time-lapse sequence before different treatments (see Methods). Each condition was repeated at least three times from different rounds of cultures and was averaged from $10-20$ cells. Error bars indicate the standard deviation (SD). ${ }^{*} p<0.05$ and ${ }^{* *} p<0.005$ in comparison to the corresponding control (Student's $t$-test). d Representative images (left) and kymographs (middle) showing the movement of mitochondria before and after A $\beta$ oligomers $1 \mu \mathrm{M} 2 \mathrm{~h}$ treatment. The kymographs are produced using the segmented lines labeled on the left images. Averaged transport speeds of mitochondria after different treatments are shown in the bar graph on the right. Data are from three dishes containing around total 60-150 moving mitochondria. Error bars indicate the standard error of the mean (SEM). ${ }^{* *} p<0.005$ when compared to the respective control period (Student's $t$-test) 
these two populations of organelles, we utilized a simplified method in which the percentage of moving organelles was quantified from the 5-min time-lapse sequence (see Methods). We found that moving endo/lysosomes accounted $50.2 \% \pm 1.3 \%($ Mean \pm SD) and $51.4 \% \pm 1.9 \%$ (Mean $\pm \mathrm{SD}$ ) of the total endo/lysosomes before and after $2 \mathrm{~h}$ exposure to $\mathrm{A} \beta-\mathrm{O}(1 \mu \mathrm{M}$ total $\mathrm{A} \beta)$, respectively. However, the number of moving mitochondria dropped from $24.3 \% \pm 2.7 \%($ Mean \pm SD) to $14.4 \% \pm 0.8 \%($ Mean \pm SD) of the total mitochondria after $2 \mathrm{~h}$ exposure to $\mathrm{A} \beta-\mathrm{O}$.

To better depict the effects of $A \beta-O$ on organelle transport, we normalized the percentage of moving organelles after different treatments to that before the treatments. As shown in Fig. 1c, the inhibitory effect of $A \beta-O$ on mitochondrial transport was rapid (e.g., $30 \mathrm{~min}$ ) and dosedependent. Significant impairment of mitochondrial transport was observed at 0.04 and $1 \mu \mathrm{M}$ total $\mathrm{A} \beta$ concentrations (corresponding to $4 \mathrm{nM}$ and $100 \mathrm{nM}$ of $\mathrm{A} \beta$ oligomers) for $2 \mathrm{~h}$ and $30 \mathrm{~min}$ exposure, respectively $(p<0.005$ comparing to that before $A \beta-O$ application, Student's $t$-test). Higher doses of $\mathrm{A} \beta-\mathrm{O}$ were found to cause severe impairment of mitochondrial transport, whereas the transport of endo/lysosomes labeled by LysoTracker remained unaffected. Importantly, no effect was observed for the reverse peptide $A \beta_{42-1}$ prepared the same way. Consistent with previous findings $[22,26]$, the impairment of mitochondrial transport by $\mathrm{A} \beta-\mathrm{O}$ was abolished by inhibition of GSK3 $\beta$ using $1 \mathrm{mM} \mathrm{LiCl}$ (Additional file 1: Figure S1).

In addition to reducing the number of moving mitochondria, $\mathrm{A} \beta-\mathrm{O}$ also slowed down the moving mitochondria. Kymograph analysis showed that the average speed of moving mitochondria was reduced to about half of that of the control period (Fig. 1d). It should be noted that all of our experiments were performed on relatively high density hippocampal cultures that contain mixed populations of highly elaborated and branched axons and dendrites. As a result, it is nearly impossible to distinguish between the MT plus end-directed anterograde and minus end-directed retrograde movements in these processes. The speed reported here represents the average of mitochondrial transport in both directions and from both axonal and dendritic processes. Consistently, $\mathrm{A} \beta-\mathrm{O}$ did not significantly affect the speed of endo/ lysosomes movement. We found that the average speed of endo/lysosomes was $0.20 \pm 0.16 \mu \mathrm{m} / \mathrm{s}($ Mean $\pm \mathrm{SD})$ before and $0.21 \pm 0.20$ (Mean $\pm \mathrm{SD}) \mu \mathrm{m} / \mathrm{s}$ after $\mathrm{A} \beta-\mathrm{O}$ treatment. Together, these results indicate that transport impairment of $\mathrm{A} \beta-\mathrm{O}$ on mitochondria was not a result of non-specific and gross disruption of the transport machinery, and may involve specific signaling pathways that target mitochondria.

We next tested if $A \beta$ oligomers from human brain tissues of Alzheimer's patients exert similar acute and selective inhibition on mitochondrial transport. Soluble fractions of frontal cortex homogenates from three AD and three age- and gender-matched control brain samples were tested in this study. Western blotting confirmed the presence of $\mathrm{A} \beta$ oligomers in $\mathrm{AD}$ samples, of which dimers appear to be the predominant form in all three AD brain samples (Fig. 2a). Consistent with our results from synthetic $A \beta-O$, all three $A D$ soluble homogenate, not that of control brain tissues, potently inhibited the transport of mitochondria without affecting the movement of endo/lysosomes (Fig. 2b). Similarly, the transport impairment of mitochondria was alleviated by $\mathrm{LiCl}$, suggesting the involvement of GSK3 $\beta$ (Additional file 1: Figure S2). To determine if the effect of soluble AD homogenates on mitochondrial transport was indeed due to the $A \beta$ molecules, we depleted $A \beta$ molecules from the homogenates by immunoprecipitation using an $A \beta$ antibody. As shown in the western blot (Fig. 2c), the beads effectively brought down $A \beta$ monomers, small oligomers, and other aggregates after merely one round (see the Output 1st). The through solution (Throu 1st) contained essentially no $A \beta$ molecules. When tested on hippocampal neurons, the through solution (Throu $1^{\text {st }}$ ) produced no effect on mitochondrial transport (Fig. 2d). Therefore, the inhibition of mitochondrial transport by $\mathrm{AD}$ soluble homogenates was caused by $A \beta$ molecules. Together, these results indicate that acute impairment of mitochondrial transport may represent one of aberrant actions of $A \beta$ oligomers on neurons, which could contribute to the disruption of neuronal functions.

Neurons are polarized in their structure and function, as highlighted by their long axonal projection and elaborated dendritic branches. Mitochondria in axon and dendrite display different morphologies and patterns of movement which may due to their different functions. We next examined if $A \beta$ impairment of mitochondrial transport is different for axonal and dendritic compartments. We expressed synaptophysin-YFP and mito-DsRed in hippocampal neurons for imaging. The axon and dendrite were distinguished by their morphology in combination with the accumulation of synaptophysin-YFP. Axons are much more elongated and thinner in comparison to the dendrites and highlighted by enriched synaptophysinYFP signals when expressed at low levels (Fig. 3a). Similar time-lapse imaging was performed before and after $A \beta-O$ application and the numbers of moving mitochondria in axonal and dendritic processes were quantified (Fig. 3b). We found that the $\mathrm{A} \beta-\mathrm{O}$ caused more potent inhibition on mitochondrial transport in dendrites than that in axonal processes $(p<0.005$, Student's $t$-test; Fig. $3 \mathrm{~b})$. We also analyzed the movement speed of mitochondria and found that it was similarly reduced in axons and dendrites (Fig. 3c), although the reduction in dendrites appears to be larger than that in axons ( $50 \%$ vs. $30 \%, p=0.035$ 

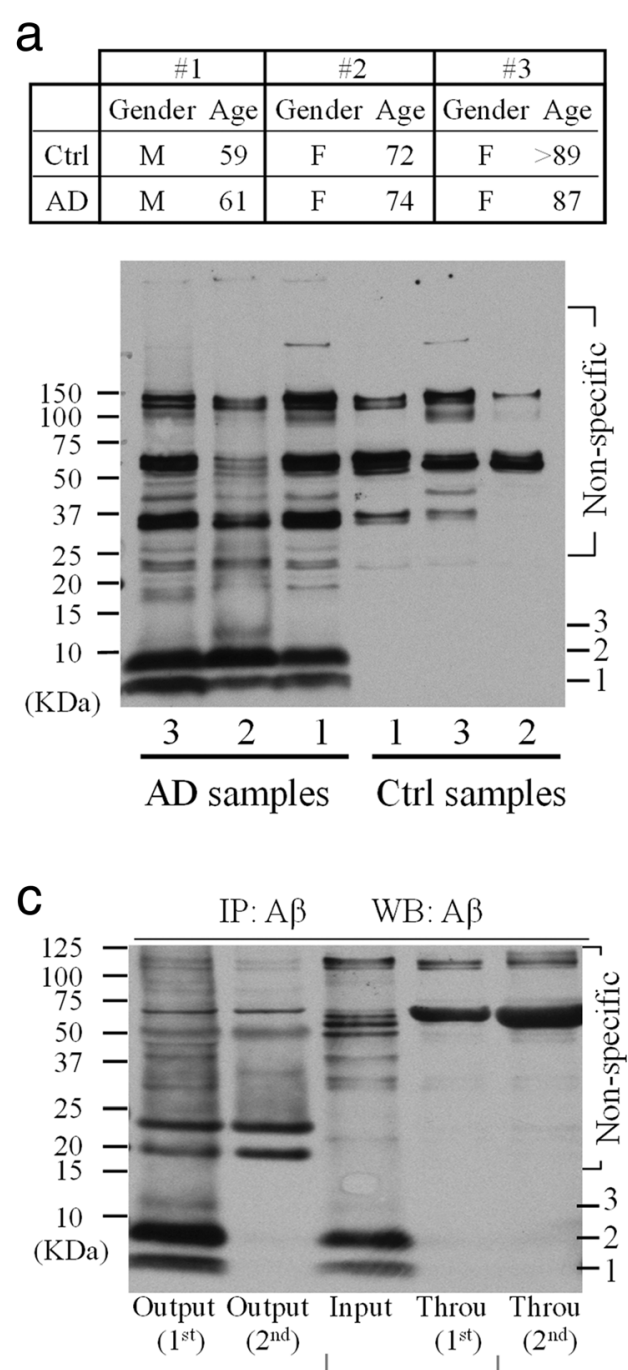

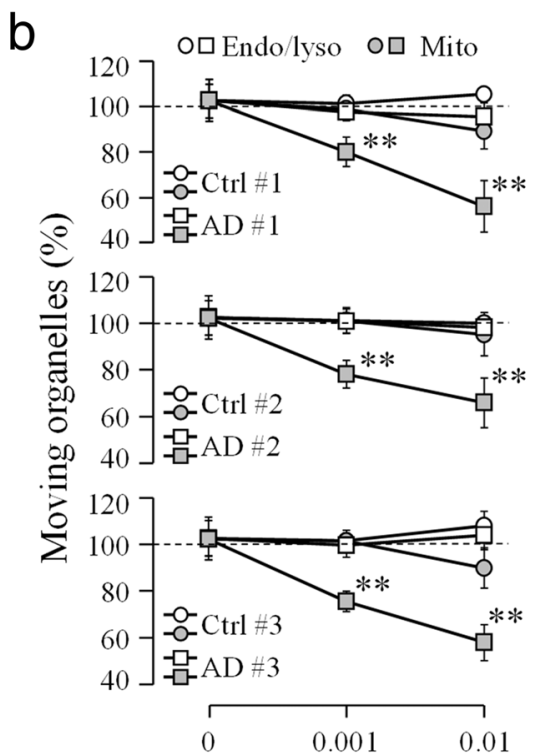

Soluble homogenate (\%)

d

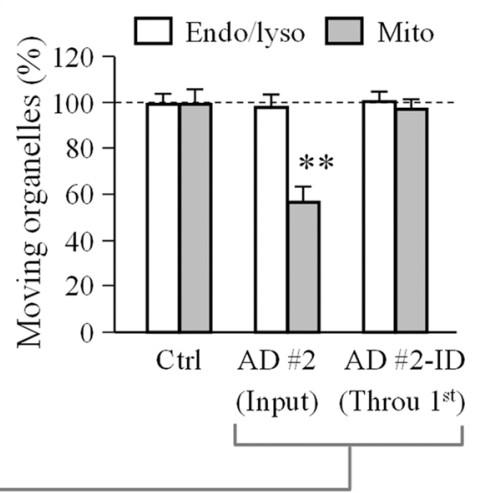

Fig. 2 Selective inhibition of fast transport of mitochondria by A $\beta$ oligomers from human brain tissues. a Western blots (lower) showing the presence of small $A \beta$ oligomers in the soluble fraction of brain homogenates from the three age- and sex-matched AD patients and control patients as indicated in the table above. A $\beta$ monomers (1) and dimmers (2) were clearly seen, whereas trimmers (3) were only seen in one AD sample. b Quantitative analysis showing the selective impairment of mitochondrial transport by different dilutions of soluble AD homogenates in KRB. c Western blots showing the effective depletion of A $\beta$ molecules by immunoprecipitation (IP). Two runs of IP were performed and the supernatants (Throu $1^{\text {st }}$ and $2^{\text {nd }}$ ) and pellets (Output $1^{\text {st }}$ and $2^{\text {nd }}$ ) were blotted. $\mathbf{d}$ Quantitative analysis showing the effects of the soluble AD brain homogenate (AD\#2, Input) and its supernatant after immunoprecipitation (Throu $1^{\text {st }}$ ) on mitochondrial transport. Error bars are SD. ${ }^{*} p<0.05$ and ${ }^{* *} p<0.005$ (Student's $t$-test). Ctrl: control; AD, AD brain sample, Throu: supernatant through IP; Mito: mitochondria; Endo/lyso: late endosomes/lysosomes. Each condition was repeated at least three times from different rounds of cultures and was averaged from 10-20 cells

Student's $t$-test). Given that the dendritic branches of a typical excitatory neuron receive and integrate a large number of synaptic inputs, $A \beta$ impairment of mitochondrial trafficking in dendrites could play a crucial part in $\mathrm{A} \beta$ disruption of synaptic functions [19].

\section{Extended exposure to $A \beta-O$ leads to mitochondrial fragmentation}

While the rapid $A \beta$ impairment of mitochondrial transport was not associated with any obvious changes in mitochondrial morphology, extended exposure to $A \beta$ oligomers was found to cause mitochondrial fragmentation (Fig. 4). When the hippocampal neurons were exposed to $5 \mu \mathrm{M}$ total $\mathrm{A} \beta$ ( $\sim 500 \mathrm{nM}$ oligomers) for $2 \mathrm{~h}$, some long mitochondria were found to break into shorter pieces (arrows in the top panels of Fig. 4a). Time-lapse imaging sequences that followed individual mitochondria showed clearly the fragmentation process (Fig. 4b; $25 \mu \mathrm{M}$ total $A \beta)$. Since fragmentation results in shorter mitochondria, we assessed the fragmentation by measuring the 


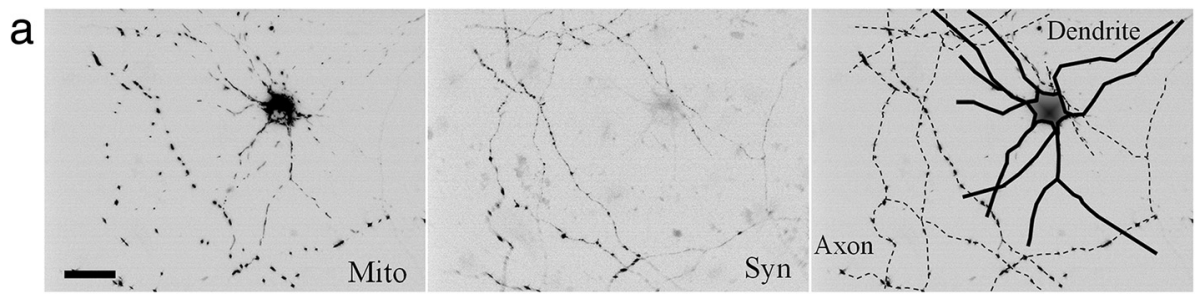

b
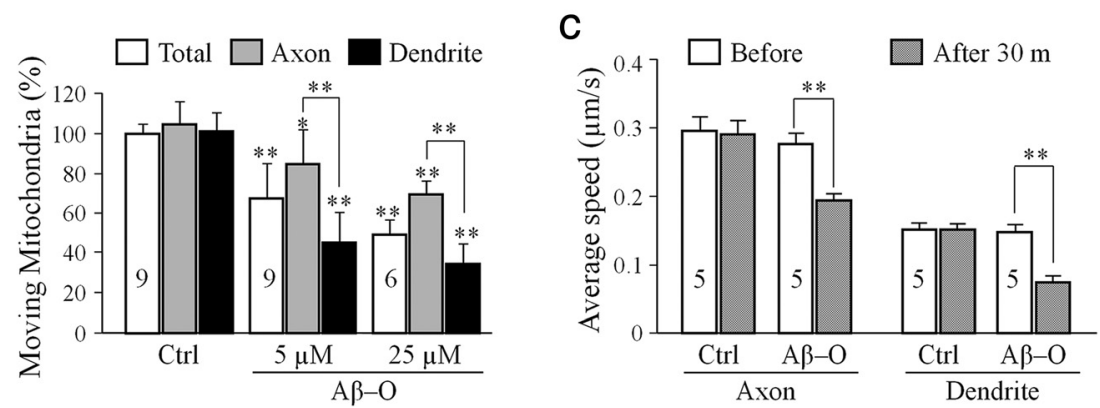

Fig. 3 Acute impairment of mitochondrial transport in axons versus dendrites. a Representative images of a DIV6 hippocampal neuron expressing Mito-DsRed (Mito, left panel) and Synaptophysin-YFP (Syn, middle panel). Both images were used to identify the axonal and dendritic compartments of the neuron, as highlighted by the schematic drawing shown in the right panel. Here, solid lines represent the dendrites and dashed lines represent the axon. Scale bars: $20 \mu \mathrm{m}$. $\mathbf{b}$ and $\mathbf{c}$ Bar graphs showing the quantitative results on the acute impairment of mitochondrial transport in number (b) and in speed (c) by $\mathrm{A} \beta-\mathrm{O}$ in axons and dendrites. Numbers indicate the number of neurons analyzed in each group (from at least three batches of culture). Error bars indicate SD (b) and SEM (c). ${ }^{*} p<0.05$ and ${ }^{* *} p<0.005$ in comparison to the correspondent control or between axons and dendrites (Student's t-test)

length of hundreds of mitochondria. We found that mitochondrial fragmentation was only observed when the cells were exposed to $5 \mu \mathrm{M}$ (total) $\mathrm{A} \beta-\mathrm{O}$ for $2 \mathrm{~h}$ or $25 \mu \mathrm{M}$ (total) $\mathrm{A} \beta-\mathrm{O}$ for $30 \mathrm{~min}$, as evidenced by the reduction in the average length of mitochondria (Fig. 4c). Consistently, $\mathrm{A} \beta$-induced fragmentation increased the total number of mitochondria (Fig. 4c). Since not all the mitochondria exhibited fragmentation upon prolonged $\mathrm{A} \beta-\mathrm{O}$ exposure, we presented the mitochondrial length of different groups in the box-whisker plot (Fig. 4d). These data show that $A \beta$ oligomers caused mitochondrial fragmentation only at a higher dose than that causing transport impairment. Therefore, $A \beta$-induced mitochondrial fragmentation is unlikely the cause of transport impairment, but a separate effect induced by $A \beta$ oligomers. Similar to $A \beta$ impairment of mitochondrial transport, mitochondrial fragmentation induced by $A \beta$ oligomers could also be abolished by inhibiting GSK3 $\beta$ with either $\mathrm{LiCl}$ or SB compounds (Additional file 1: Figure S3), supporting a central role for GSK3 $\beta$ in mediating $A \beta$ effects.

\section{Distinct $A \beta$ effects on mitochondria are mediated by specific pathways}

We next investigated how $\mathrm{A} \beta$ oligomers elicit transport impairment and fragmentation of mitochondria, two effects with distinct time courses and dose-dependence. We first examined if prolonged $\mathrm{A} \beta-\mathrm{O}$ exposure may disrupt the mitochondrial membrane potential leading to transport impairment and fragmentation. Live cell imaging using the TMRE dye $[27,28]$ showed that $2 \mathrm{~h}$ exposure to $5 \mu \mathrm{M}$ (total concentration) $\mathrm{A} \beta-\mathrm{O}$ did not affect the TMRE signal (Additional file 1: Figure S4). We next examined if $A \beta$ oligomers caused cell death, which in turn resulted in mitochondrial transport defects and fragmentation. The cell viability assay [29] showed that cell death only started to be observed after $6 \mathrm{~h}$ exposure to A $\beta-O$ (Additional file 1: Figure S5a). Similar results were also obtained using a different method in which a Hoechst dye was used to identify apoptotic nuclei (Additional file 1: Figure S5b). The very late onset of cell death upon $\mathrm{A} \beta-\mathrm{O}$ exposure argues against cell death as the cause of the acute mitochondria defects induced by $\mathrm{A} \beta$ oligomers.

Recent studies have implicated the cytoplasmic histone deacetylase 6 (HDAC6) in several neurodegenerative diseases including Alzheimer's, Parkinson's and Huntington's diseases [30, 31]. Moreover, HDAC6 has been shown to regulate mitochondrial transport in hippocampal neurons [32]. We found that although acute exposure of hippocampal neurons to $A \beta$ oligomers did not affect the expression of HDAC6 (Additional file 1: Figure S6 a and b), it did appear to increase the activities of HDACs in the cytosol and mitochondria as measured biochemically using the HDAC activity fluorometric assay kit (Additional file 1: Figure S6c). Since HDAC6 is the major HDAC in the cytoplasm, this result suggests that 


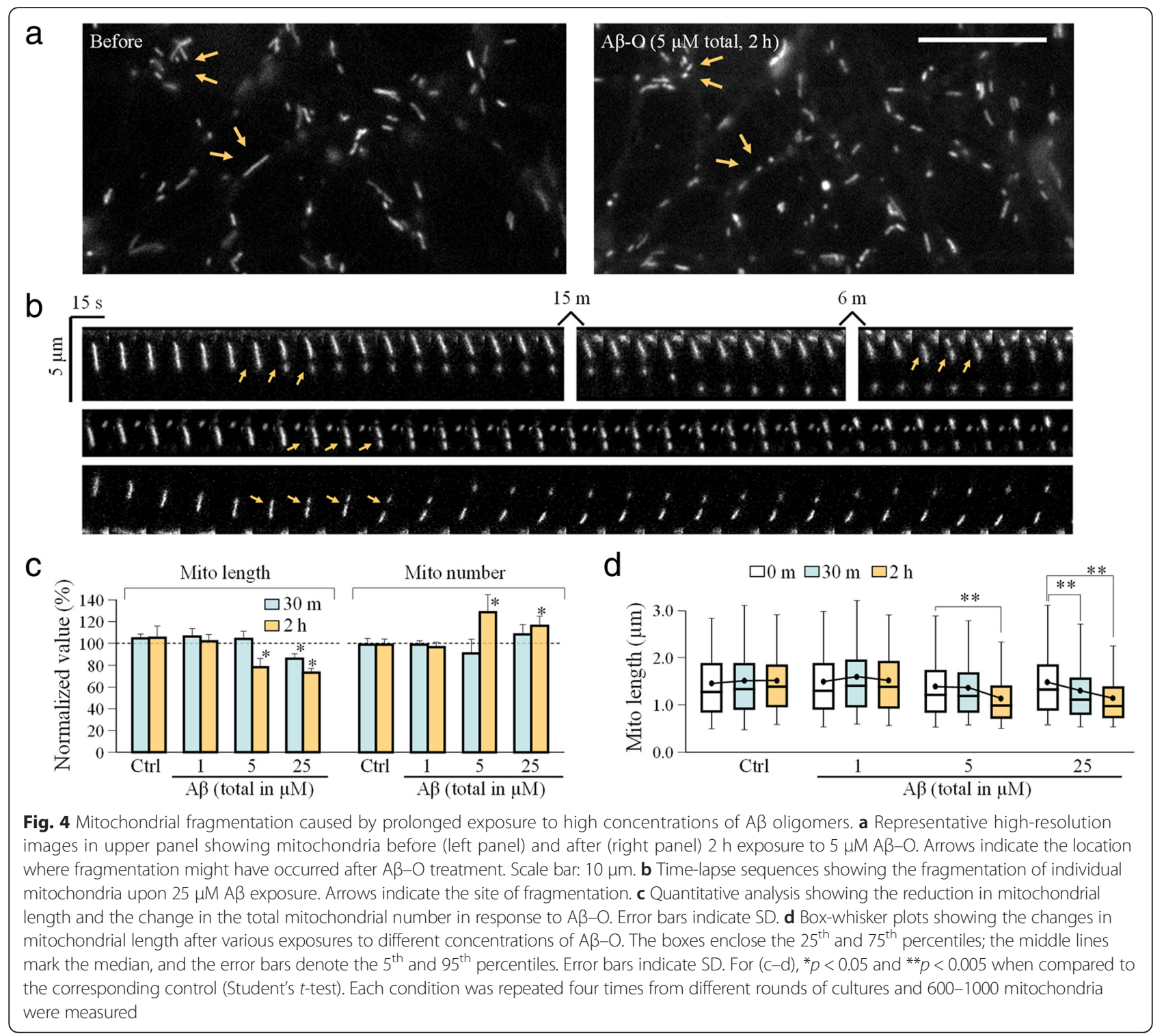

A $\beta$ oligomers elevated the HDAC6 activities, which is supported by increased deacetylation of $\alpha$-tubulin after $A \beta-O$ exposure (Additional file 1: Figure S6 d-f). We thus tested if inhibition of HDAC6 could alleviate the A $\beta$ effects on mitochondrial transport and structure. Application of trichostatin A (TSA), a selective inhibitor for the class I and II HDACs that includes HDAC6, completely abolished the acute impairment of mitochondrial transport by $A \beta-O$ (Fig. 5a). We further tested tubacin, a specific HDAC6 inhibitor [33], and MS-275, a selective HDAC1 inhibitor [34] and found that only tubacin was able to abolish $A \beta$ impairment of mitochondrial transport (Fig. 5a). Our kymograph analysis also showed that inhibition of HDAC6 by TSA or tubacin effectively arrogated $A \beta$-induced reduction in the transport speed of mitochondria (Additional file 1: Figure S7). Interestingly, neither TSA nor tubacin affected the mitochondrial fragmentation induced by $\mathrm{A} \beta$ $\mathrm{O}$ (Fig. $5 \mathrm{~b}$ and $\mathrm{c}$ ). Since mitochondria undergo fusion and fission, the latter is mediated by the dynaminrelated GTPase (Drp1). We examined if mitochondria fission was involved in $\mathrm{A} \beta$-induced fragmentation. We found that inhibition of Drp1 by the selective inhibitor mdivi-1 blocked $\mathrm{A} \beta$-induced mitochondrial fragmentation (Fig. $5 \mathrm{~b}$ and c). However, mdivi-1 had no effect on the transport defects elicited by $\mathrm{A} \beta-\mathrm{O}$ (Fig. $5 \mathrm{a}$ ). Taken together, these results suggest that $A \beta$ oligomers exert timedependent and pathway-specific effects on mitochondrial transport and structure.

\section{Discussion}

Stalled and deformed mitochondria in clusters are one of the outstanding signs of many neurodegenerative 


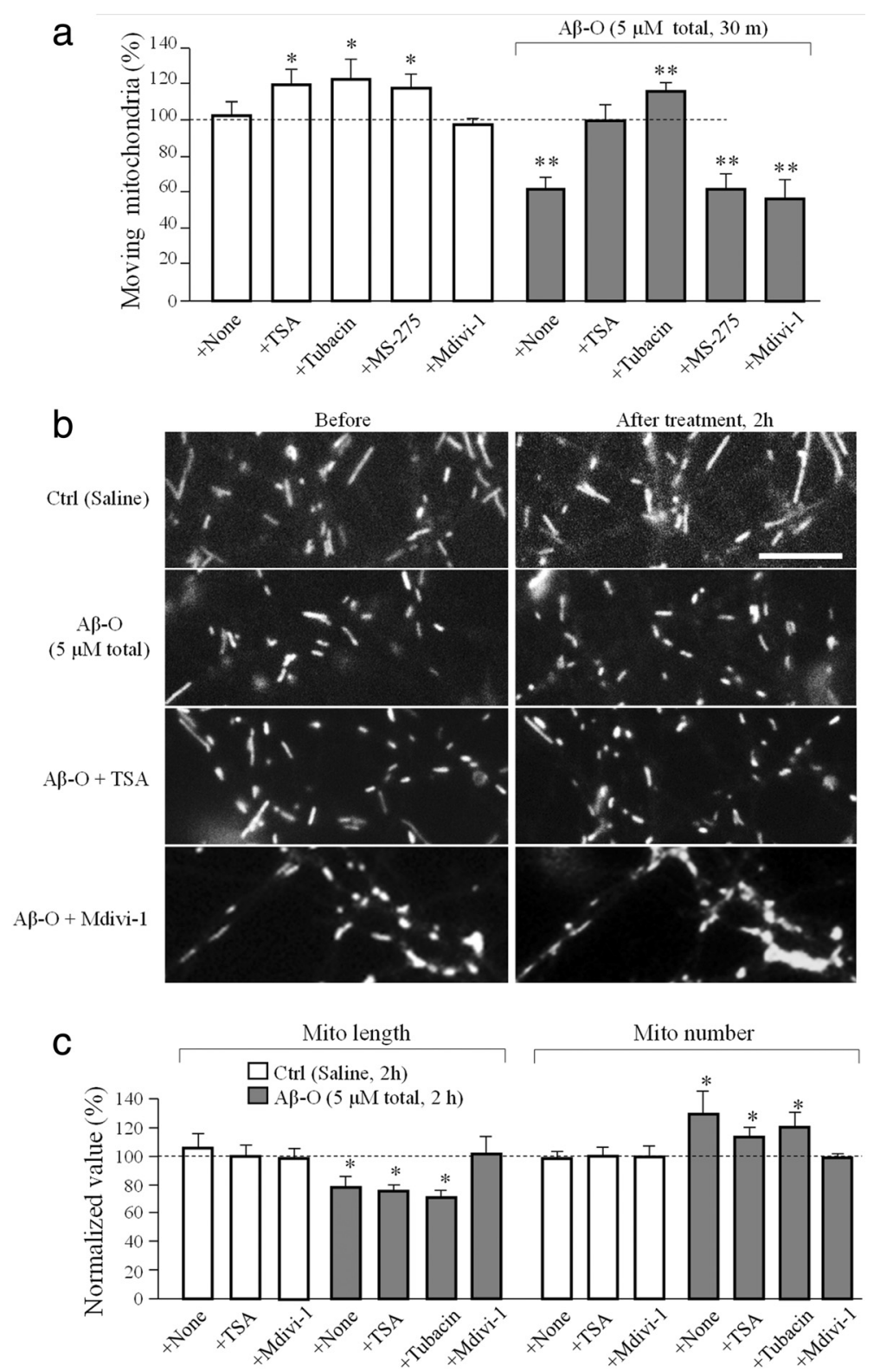

Fig. 5 Effect of HDAC6 or Drp1 inhibition on A $3-\mathrm{O}$ induced mitochondria trafficking impairment and fragmentation. a Quantitative analysis showing the changes in moving mitochondria after 30 min exposure to different HDAC inhibitors (TSA $10 \mu \mathrm{M}$, tubacin $20 \mu \mathrm{M}, \mathrm{MS}-2755 \mu \mathrm{M}$ ) or Drp1 inhibitor (Mdivi-1 $20 \mu \mathrm{M}$ ) with and without A $-\mathrm{O}$. b Representative images showing the morphology of mitochondria before and after $2 \mathrm{~h}$ exposure to control or AB-O (5 $\mu \mathrm{M}$ total) without or with presence of TSA or Mdivi-1. Scale bar: $5 \mu \mathrm{m}$. c Bar graph showing the changes in the average mitochondrial length and total number in response to A $\beta-O$ without and with HDAC6 or Drp1 inhibitions in bath. Numbers represent the number of dishes examined with a total of 10-20 cells for each data point. Error bars indicate SD. ${ }^{*} p<0.05$ and ${ }^{* *} p<0.005$ in comparison to the corresponding control (Student's $t$ test)

diseases [35, 36]. In $\mathrm{AD}$, disruption in mitochondrial transport and function contributes to the disease pathogenesis and brain dysfunction [37-39]. While $A \beta$ plaques are a hallmark of degenerating AD brains, soluble $A \beta$ oligomers are believed to be the culprit underlying a variety of toxic effects on neuronal functions leading to the cognitive decline of the AD brain. While the adverse effects of $A \beta$ oligomers on neurons and synapses are diverse and involve different targets, mitochondria are one of the major targets that $A \beta$ oligomers negatively impact. In this study, we present evidence that $A \beta$ oligomers elicit two acute effects on mitochondria: impairment of fast transport and fragmentation. Importantly we show that these two acute effects of $A \beta$ oligomers involve different exposure times and doses of $A \beta$ oligomer and are mediated by distinct signaling pathways. The 
selective impairment of mitochondrial transport is rapidly induced by low concentrations of by $\mathrm{A} \beta$ oligomers, whereas the fragmentation requires longer exposure and higher doses. While both $A \beta$ effects on mitochondria can be blocked by inhibition of GSK3 $\beta$, the transport impairment requires the activation of HDAC6 and fragmentation involves the GTPase drp1. Given that fast transport and fusion/fission of mitochondria are crucial for their function at subcellular locations, impairment of mitochondria transport and disruption of fusion/fission balance could argument the local function of mitochondria, such as at synapses.

Acute impairment of mitochondrial transport by $A \beta$ molecules has been reported previously [22, 40]. This study has not only substantiated previous findings, but also provided additional insights. First, our findings show that $A \beta$ oligomers selectively impair mitochondrial transport without affecting that of lysosomes and endosomes. Second, we present the evidence that $A \beta$ oligomers from human $\mathrm{AD}$ brains exert the similar impairment of mitochondria transport to that of synthetic $A \beta$ oligomers, indicating that this effect could potentially occur in human brains. Finally, our data suggest that $A \beta$ oligomers negatively impact mitochondrial transport more effectively in dendrites than in axons. Mitochondria in axonal and dendritic compartments display different morphology and motility that may reflect their difference in functions in these two compartments [41]. The long and slender axons of neurons contain a limited space that is prone to road blocks and traffic jams for long range transport, especially under neurodegenerative conditions [17]. Dendrites, on the other hand, are shorter and thicker but form more elaborated arbors that function in receiving and integrating hundreds of synaptic inputs. Trafficking of synaptic receptors, mitochondria and other organelles, and mRNA-ribosomal complex occur actively in dendritic compartments and play a crucial role in postsynaptic functions [6, 10, 42, 43]. Our finding that mitochondrial transport in dendrites appears to be more severely inhibited by $A \beta$ oligomers is consistent with the emerging view of $A \beta$ adverse effects on postsynaptic structure and function $[19,44,45]$. Disrupted mitochondrial transport in dendrites could negatively impact the distribution and health of mitochondria to affect a number of mitochondria-dependent postsynaptic events, such as $\mathrm{Ca}^{2+}$ signaling, receptor trafficking, spine structure and plasticity, leading to synaptic dysfunction and degeneration [10].

The signaling mechanisms underlying the acute effects of $A \beta$ oligomers on mitochondria remain to be elucidated. While inhibition of GSK3 $\beta$ appears to alleviate both $A \beta$ effects, the transport impairment and fragmentation of mitochondria induced by $\mathrm{A} \beta$ oligomers are selectively abolished by the inhibition of HDAC6 and Drp1, respectively. HDAC6 is a member of class II HDACs that is localized mostly in the cytoplasm with a preference for non-histone proteins [46]. Increased HDAC6 level in cortex and hippocampus of AD brain has been reported [47]. HDAC6 has been found to deacetylate multiple non-histone proteins [48] such as $\alpha$-tubulin [46, 49], tau [50], HSP90 [51] and cortactin [49]. The lysine 40 (K40) of $\alpha$-tubulin is posttranslationally modified through acetylation in cells and HDAC6 is known to be the main deacetylase for this site, but this posttranslational modification does not appear to affect microtubule motor-based transport [52-54]. Therefore,

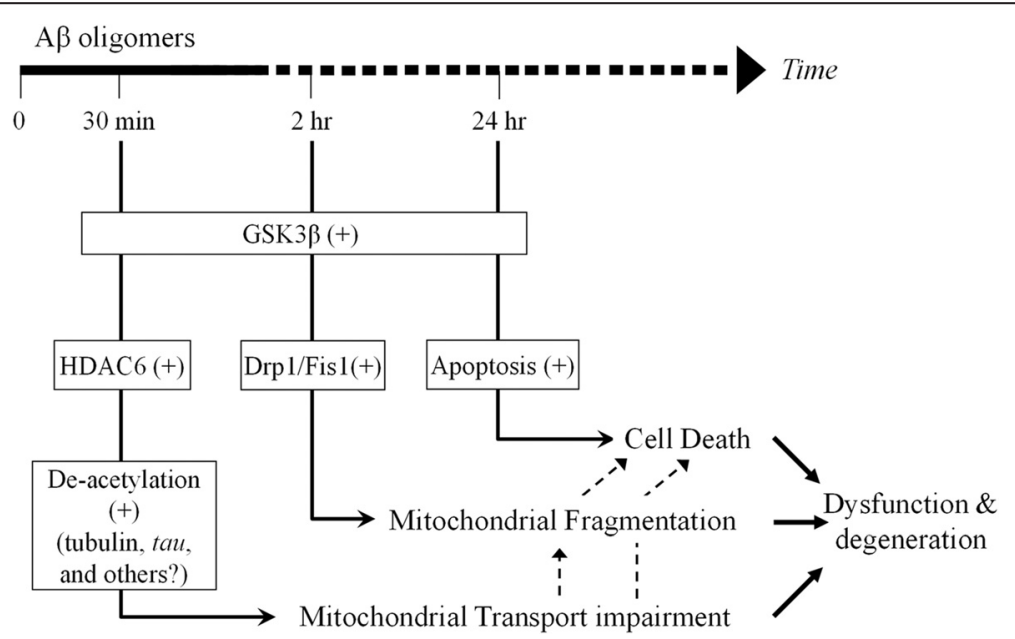

Fig. 6 A schematic showing the temporal segregation and potential involvement of different pathways among three adverse effects of $A \beta$ oligomers: mitochondrial transport impairment, fragmentation, and cell death. All three effects appear to involve GSK3 $\beta$. A $\beta$ impairment of mitochondrial transport involved HDAC6-mediated microtubule deacetylation. Mitochondrial fragmentation may involve the mitochondrial fission components of Drp1 and Fis1, whereas cell death is mediated by the apoptosis pathway. Dashed lines indicate potential alternative pathways 
other potential targets of HDAC6, such as tau [55], need to be investigated for their role in $A \beta$ impairment of mitochondrial transport.

Our data suggest that the fragmentation induced by A $\beta$ oligomers involves Drp1, a GTPase involved in mitochondria fission. Mitochondria are known to undergo fission and fusion, which are highly regulated to control the cellular distribution, health state, and function of mitochondria [56]. The $A \beta$ targeting of Drp1 can result in excessive fission of mitochondria, leading to the disruption of mitochondrial distribution and function. Indeed altered expression levels of proteins involved in mitochondria fission and fusion have been observed in AD brains [57]. How A $\beta$ oligomers activate Drp1 remains unknown at this moment. Drp1 can be regulated by a number of posttranslational modifications including phosphorylation [58], SUMOylation [59] and S-Nirosylation [60]. S-Nirosylation of Drp1 is increased in brain of human AD patients and inhibition of S-nitrosylation of Drp1 was shown to abrogate $A \beta$ triggered mitochondria fission, synaptic loss and neuronal damage [60]. On the other hand, GSK3 $\beta$ was found to interact with Drp1 to induce Drp1 phosphorylation, leading to mitochondria fragmentation in $\mathrm{AD}[61,62]$. Given that GSK3 $\beta$ has been shown to regulate HDAC6 activity [37], GSK3 $\beta$ could acts as a central node to transmit a wide range of $A \beta$ actions, including HDAC6 activation and Drp1 phosphorylation to impact mitochondrial transport and mitochondrial fragmentation (Fig. 6). The next challenge is to elucidate how the signal divergence from GSK3 $\beta$ is achieved and regulated to generate distinct cellular effects underlying detrimental actions of $A \beta$ oligomers.

\section{Additional file}

Additional file 1: Supplemental figures S1-S7 and methods. (PDF 902 kb)

\section{Acknowledgements}

We would like to thank Drs. Jason Fritz and Marla Gearing at Emory CND for their help with human tissue samples. We also thank Dr. Stuart L. Schreiber from Howard Hughes Medical Institute for providing us the specific HDAC6 inhibitor tubacin.

\section{Funding}

This research was supported in part by grants from the National Institutes of Health (GM083889 and MH104632), a pilot grant from Emory Alzheimer's Disease Resource Center (ADRC P50 AG025688), a NINDS core facilities grant (P30NS055077) to the Neuronal Imaging Core of Emory Neuroscience, and a postdoctoral fellowship from the Ellison Medical Foundation/American Federation for Aging Research to YR.

\section{Availability of data and materials}

Not applicable.

\section{Authors' contributions}

YR designed and performed all the experiments and drafted the manuscript. JQZ oversaw and guided the study and wrote the manuscript together with YR. Both authors read and approved the final manuscript.

\section{Competing interests}

The authors declare that they have no competing interests.

\section{Consent for publication}

Not applicable.

\section{Ethics approval and consent to participate}

All the experiments involving the use of vertebrate animals were carried out in accordance to the $\mathrm{NIH}$ guideline for animal use and have been approved by the Institutional Animal Care and Use Committee of Emory University. No human subjects were included in this study.

\section{Author details}

'Department of Cell Biology, Emory University School of Medicine, 615 Michael Street, Atlanta, GA 30322, USA. ²Department of Neurology, Emory University School of Medicine, Atlanta, GA 30322, USA. ${ }^{3}$ Center for Neurodegenerative Diseases, Emory University School of Medicine, Atlanta, GA 30322, USA.

Received: 11 July 2016 Accepted: 11 August 2016

Published online: 17 August 2016

\section{References}

1. Glenner GG, Wong CW. Alzheimer's disease: initial report of the purification and characterization of a novel cerebrovascular amyloid protein. Biochem Biophys Res Commun. 1984;120:885-90.

2. Walsh DM, Selkoe DJ. A beta oligomers - a decade of discovery. J Neurochem. 2007:101:1172-84

3. Krafft GA, Klein WL. ADDLs and the signaling web that leads to Alzheimer's disease. Neuropharmacology. 2010:59:230-42.

4. Haass C, Selkoe DJ. Soluble protein oligomers in neurodegeneration: lessons from the Alzheimer's amyloid beta-peptide. Nat Rev Mol Cell Biol. 2007;8:101-12.

5. Reddy PH, Beal MF. Amyloid beta, mitochondrial dysfunction and synaptic damage: implications for cognitive decline in aging and Alzheimer's disease. Trends Mol Med. 2008;14:45-53.

6. MacAskill AF, Kittler JT. Control of mitochondrial transport and localization in neurons. Trends Cell Biol. 2010;20:102-12.

7. Hollenbeck PJ, Saxton WM. The axonal transport of mitochondria. J Cell Sci. 2005;118:5411-9.

8. Verstreken P, Ly CV, Venken KJ, Koh TW, Zhou Y, et al. Synaptic mitochondria are critical for mobilization of reserve pool vesicles at Drosophila neuromuscular junctions. Neuron. 2005;47:365-78.

9. Li Z, Okamoto K, Hayashi Y, Sheng M. The importance of dendritic mitochondria in the morphogenesis and plasticity of spines and synapses. Cell. 2004;119:873-87.

10. Mattson MP, Gleichmann M, Cheng A. Mitochondria in neuroplasticity and neurological disorders. Neuron. 2008;60:748-66.

11. Chang DT, Honick AS, Reynolds IJ. Mitochondrial trafficking to synapses in cultured primary cortical neurons. J Neurosci. 2006;26:7035-45.

12. Guo X, Macleod GT, Wellington A, Hu F, Panchumarthi S, et al. The GTPase dMiro is required for axonal transport of mitochondria to Drosophila synapses. Neuron. 2005:47:379-93.

13. Westermann B. Mitochondrial fusion and fission in cell life and death. Nat Rev Mol Cell Biol. 2010;11:872-84

14. Diaz F, Moraes CT. Mitochondrial biogenesis and turnover. Cell Calcium. 2008:44:24-35.

15. Schon EA, Przedborski S. Mitochondria: the next (neurode)generation. Neuron. 2011;70:1033-53.

16. Stokin GB, Goldstein LS. Axonal transport and Alzheimer's disease. Annu Rev Biochem. 2006;75:607-27.

17. De Vos KJ, Grierson AJ, Ackerley S, Miller CC. Role of axonal transport in neurodegenerative diseases. Annu Rev Neurosci. 2008;31:151-73.

18. Du H, Guo L, Yan S, Sosunov AA, McKhann GM, et al. Early deficits in synaptic mitochondria in an Alzheimer's disease mouse model. Proc Natl Acad Sci U S A. 2010;107(43):18670-18675.

19. Rui Y, Gu J, Yu K, Hartzell HC, Zheng JQ. Inhibition of AMPA receptor trafficking at hippocampal synapses by beta-amyloid oligomers: the mitochondrial contribution. Mol Brain. 2010;3:10.

20. Banker GA, Cowan WM. Rat hippocampal neurons in dispersed cell culture. Brain Res. 1977;126:397-425. 
21. Bacci A, Verderio C, Pravettoni E, Matteoli M. Synaptic and intrinsic mechanisms shape synchronous oscillations in hippocampal neurons in culture. Eur J Neurosci. 1999;11:389-97.

22. Rui $Y$, Tiwari $P$, Xie Z, Zheng JQ. Acute impairment of mitochondrial trafficking by beta-amyloid peptides in hippocampal neurons. J Neurosci. 2006;26:10480-7.

23. Dahlgren KN, Manelli AM, Stine Jr WB, Baker LK, Krafft GA, et al. Oligomeric and fibrillar species of amyloid-beta peptides differentially affect neuronal viability. J Biol Chem. 2002;277:32046-53.

24. Mirra SS, Heyman A, McKeel D, Sumi SM, Crain BJ, et al. The Consortium to Establish a Registry for Alzheimer's Disease (CERAD). Part II. Standardization of the neuropathologic assessment of Alzheimer's disease. Neurology. 1991:41:479-86.

25. Hyman BT, Trojanowski JQ. Consensus recommendations for the postmortem diagnosis of Alzheimer disease from the National Institute on Aging and the Reagan Institute Working Group on diagnostic criteria for the neuropathological assessment of Alzheimer disease. J Neuropathol Exp Neurol. 1997:56:1095-7.

26. Decker H, Lo KY, Unger SM, Ferreira ST, Silverman MA. Amyloid-beta peptide oligomers disrupt axonal transport through an NMDA receptordependent mechanism that is mediated by glycogen synthase kinase 3 beta in primary cultured hippocampal neurons. J Neurosci. 2010;30:9166-71.

27. Nicholls DG, Budd SL. Mitochondria and neuronal survival. Physiol Rev. 2000;80:315-60

28. Loew LM, Tuft RA, Carrington W, Fay FS. Imaging in five dimensions: timedependent membrane potentials in individual mitochondria. Biophys J. 1993;65:2396-407.

29. Vaughan PJ, Pike CJ, Cotman CW, Cunningham DD. Thrombin receptor activation protects neurons and astrocytes from cell death produced by environmental insults. J Neurosci. 1995;15:5389-401.

30. Simoes-Pires C, Zwick V, Nurisso A, Schenker E, Carrupt PA, et al. HDAC6 as a target for neurodegenerative diseases: what makes it different from the other HDACs? Mol Neurodegener. 2013;8:7. doi:10.1186/1750-1326-8-7.

31. Govindarajan N, Rao P, Burkhardt S, Sananbenesi F, Schluter OM, et al. Reducing HDAC6 ameliorates cognitive deficits in a mouse model for Alzheimer's disease. EMBO Mol Med. 2013;5:52-63.

32. Chen SG, Owens GC, Makarenkova H, Edelman DB. HDAC6 regulates mitochondrial transport in hippocampal neurons. PLoS One. 2010;5(5): e10848. doi:10.1371/journal.pone.0010848.

33. Haggarty SJ, Koeller KM, Wong JC, Grozinger CM, Schreiber SL. Domainselective small-molecule inhibitor of histone deacetylase 6 (HDAC6)-mediated tubulin deacetylation. Proc Natl Acad Sci U S A. 2003;100:4389-94.

34. Suzuki T, Ando T, Tsuchiya K, Fukazawa N, Saito A, et al. Synthesis and histone deacetylase inhibitory activity of new benzamide derivatives. J Med Chem. 1999:42:3001-3.

35. Johri A, Beal MF. Mitochondrial dysfunction in neurodegenerative diseases. J Pharmacol Exp Ther. 2012;342:619-30.

36. Chen $\mathrm{H}$, Chan DC. Mitochondrial dynamics-fusion, fission, movement, and mitophagy-in neurodegenerative diseases. Hum Mol Genet. 2009;18:R169-76.

37. Reddy PH, Beal MF. Are mitochondria critical in the pathogenesis of Alzheimer's disease? Brain Res Brain Res Rev. 2005;49:618-32.

38. Moreira PI, Carvalho C, Zhu XW, Smith MA, Perry G. Mitochondrial dysfunction is a trigger of Alzheimer's disease pathophysiology. Biochim Biophys Acta Mol Basis Dis. 2010;1802:2-10.

39. Swerdlow RH, Burns JM, Khan SM. The Alzheimer's disease mitochondrial cascade hypothesis: progress and perspectives. Biochim Biophys Acta. 2014;1842:1219-31.

40. Decker H, Lo KY, Unger SM, Ferreira ST, Silverman MA. Amyloid-beta peptide oligomers disrupt axonal transport through an NMDA receptordependent mechanism that is mediated by glycogen synthase kinase 3 beta in primary cultured hippocampal neurons. J Neurosci. 2010;30:9166-71.

41. Overly CC, Rieff HI, Hollenbeck PJ. Organelle motility and metabolism in axons vs dendrites of cultured hippocampal neurons. J Cell Sci. 1996;109(Pt 5):971-80.

42. Ramirez OA, Couve A. The endoplasmic reticulum and protein trafficking in dendrites and axons. Trends Cell Biol. 2011;21:219-27.

43. Martin KC, Zukin RS. RNA trafficking and local protein synthesis in dendrites: an overview. J Neurosci. 2006:26:7131-4

44. Hsieh H, Boehm J, Sato C, Iwatsubo T, Tomita T, et al. AMPAR removal underlies Abeta-induced synaptic depression and dendritic spine loss. Neuron. 2006;52:831-43.
45. Renner M, Lacor PN, Velasco PT, Xu J, Contractor A, et al. Deleterious effects of amyloid beta oligomers acting as an extracellular scaffold for mGluR5. Neuron. 2010;66:739-54.

46. Hubbert C, Guardiola A, Shao R, Kawaguchi Y, Ito A, et al. HDAC6 is a microtubule-associated deacetylase. Nature. 2002;417:455-8.

47. Ding H, Dolan PJ, Johnson GV. Histone deacetylase 6 interacts with the microtubule-associated protein tau. J Neurochem. 2008;106:2119-30.

48. Cook C, Gendron TF, Scheffel K, Carlomagno Y, Dunmore J, et al. Loss of HDAC6, a novel CHIP substrate, alleviates abnormal tau accumulation. Hum Mol Genet. 2012;21:2936-45.

49. Ran J, Yang YF, Li DW, Liu M, Zhou J. Deacetylation of alpha-tubulin and cortactin is required for HDAC6 to trigger ciliary disassembly. Sci Rep. 2015:5:12917. doi:10.1038/srep12917.

50. Cook C, Carlomagno Y, Gendron TF, Dunmore J, Scheffel K, et al. Acetylation of the KXGS motifs in tau is a critical determinant in modulation of tau aggregation and clearance. Hum Mol Genet. 2014;23:104-16.

51. Kovacs JJ, Murphy PJ, Gaillard S, Zhao X, Wu JT, et al. HDAC6 regulates Hsp90 acetylation and chaperone-dependent activation of glucocorticoid receptor. Mol Cell. 2005:18:601-7.

52. Kaul N, Soppina V, Verhey KJ. Effects of alpha-tubulin K40 acetylation and detyrosination on kinesin-1 motility in a purified system. Biophys J. 2014;106:2636-43.

53. Walter WJ, Beranek V, Fischermeier E, Diez S. Tubulin acetylation alone does not affect kinesin-1 velocity and run length in vitro. PLoS One. 2012;7(8): e42218. doi:10.1371/journal.pone.0042218

54. Kalebic N, Sorrentino S, Perlas E, Bolasco G, Martinez C, et al. alpha TAT1 is the major alpha-tubulin acetyltransferase in mice. Nat Commun. 2013;4: 1962. doi:10.1038/ncomms2962.

55. Cook C, Stankowski JN, Carlomagno Y, Stetler C, Petrucelli L. Acetylation: a new key to unlock tau's role in neurodegeneration. Alzheimers Res Ther. 2014;6(3):29. doi:10.1186/alzrt259.

56. Chan DC. Mitochondrial fusion and fission in mammals. Annu Rev Cell Dev Biol. 2006:22:79-99.

57. Wang X, Su B, Lee HG, Li X, Perry G, et al. Impaired balance of mitochondrial fission and fusion in Alzheimer's disease. J Neurosci. 2009;29:9090-103.

58. Chang CR, Blackstone C. Drp1 phosphorylation and mitochondrial regulation. EMBO Rep. 2007;8:1088-9. author reply 1089-1090.

59. Figueroa-Romero C, Iniguez-Lluhi JA, Stadler J, Chang CR, Arnoult D, et al. SUMOylation of the mitochondrial fission protein Drp1 occurs at multiple nonconsensus sites within the $B$ domain and is linked to its activity cycle. FASEB J. 2009:23:3917-27.

60. Cho DH, Nakamura T, Fang JG, Cieplak P, Godzik A, et al. S-nitrosylation of Drp1 mediates beta-amyloid-related mitochondrial fission and neuronal injury. Science. 2009;324:102-5.

61. Kandimalla R, Reddy PH. Multiple faces of dynamin-related protein 1 and its role in Alzheimer's disease pathogenesis. Biochim Biophys Acta. 2016;1862:814-28.

62. Yan J, Liu XH, Han MZ, Wang YM, Sun XL, et al. Blockage of GSK3betamediated Drp1 phosphorylation provides neuroprotection in neuronal and mouse models of Alzheimer's disease. Neurobiol Aging. 2015;36:211-27.

\section{Submit your next manuscript to BioMed Central and we will help you at every step:}

- We accept pre-submission inquiries

- Our selector tool helps you to find the most relevant journal

- We provide round the clock customer support

- Convenient online submission

- Thorough peer review

- Inclusion in PubMed and all major indexing services

- Maximum visibility for your research

Submit your manuscript at www.biomedcentral.com/submit 